\title{
Reduction of Aspergillus spp. and aflatoxins in peanut sauce processing by oil-less frying of chilli powder and retort processing
}

\begin{abstract}
Among the many roles played by small and medium enterprises (SMEs) in the food industry is the production of heritage foods such as peanut sauce. Unfortunately, the safety of peanut sauce is not always assured as the processing line is not controlled. Peanut sauce is usually made of peanuts and chilli, and these commodities are normally contaminated with Aspergillus spp. and aflatoxins (AFs). Hence, the objective of this study was to evaluate the practices related to reduction of $\mathrm{AF}$ hazard and the effect of interventions in peanut sauce processing. Peanut samples were collected from each step of peanut sauce processing from a small peanut sauce company according to four designs: (1) control; (2) oil-less frying of chilli powder; (3) addition of retort processing; and (4) combination of oil-less frying of chilli powder and retort processing. Oil-less frying of chilli powder (Design 2) reduced total AFs by $33-41 \%$, retort processing (Design 3) reduced total AFs by $49 \%$, while combination of these two thermal processes (Design 4) significantly reduced total AFs, by 57\%. The present work demonstrated that Design 4 yielded the highest reduction of total AFs and is therefore recommended to be employed by SME companies.
\end{abstract}

Keyword: HPLC; Aflatoxins; Clean-up - affinity columns; Method validation; Mycology; Novel food; Peanuts 